\title{
Atomic Resolved Secondary Electron Imaging with an Aberration Corrected Scanning Transmission Electron Microscope
}

\author{
H. Inada, ${ }^{*}$ K. Tamura, ${ }^{*}$ K. Nakamura, ${ }^{*}$ Y. Suzuki, ${ }^{*}$ M. Konno, ${ }^{*}$ D. Su, ${ }^{* *}$ J. Wall,**, R. \\ Egerton***, and Y. Zhu** \\ * Nanotechnology Products Business Group, Hitachi High-Technologies Corp., Hitachinaka, Ibaraki \\ 312-8504 Japan \\ ** Brookhaven National Laboratory, Upton NY 11973 \\ *** University of Alberta, Alberta Canada T6G 2G7
}

For the past few years, aberration correction technology has been providing routine atomic resolution imaging and analyses capabilities to scanning transmission electron microscope (STEM) and brought a revolution in analytical microscopy. A modern scanning electron microscope (SEM), on the other hand, is widely used to observe various types of specimen surface features on scales ranging from nanometers to tens of micron. Most advanced conventional commercial SEMs achieve a spatial resolution of $0.4 \mathrm{~nm}$. The secondary electron imaging mode is commonly operated in SEMs, which allows us to observe three-dimensional topographic images of the sample surface. We have succeeded in observing isolated single uranium atoms using secondary electron signals with an aberration corrector equipped on a STEM with an acceleration voltage of $200 \mathrm{kV}$ installed at Brookhaven National Laboratory $[1,2]$. We observed atomic-resolution images not only for heavy elements like uranium but also for ight ones like silicon and carbon. The ability of atomically resolved secondaryelectron imaging is unprecedented and can lead to a new understanding of electronic signal generation for a sample surface.

We have been investigating the origin of the electrons collected by the secondary electron detectors that from the atomic images. We carried out the atomically resolved SE imaging with a $200 \mathrm{kV}$ aberration corrected STEM, Hitachi HD-2700, that has an ability of simultaneous signal acquisition for secondary electrons (SE) images, bright-field (BF) and annular dark-field (ADF) STEM images. The SE detector, which is based on an Everhart-Thornley design, is installed above the pole piece and a $10 \mathrm{kV}$ dc bias is applied to the phosphor to attract secondary electrons with the so called through the lens (TTL) design (Figure 1). We have studied lower resolution uranyl-accetate stained Tabacco Mosaic Virus on carbon thin film specimen previously and revealed that the contrast originates from the secondary electrons because the signal intensity decreases with the increase of applied positive bias. The secondary-electron trajectory calculations based on the microscope's design using Monte Carlo simulation suggests the SE detector can attract electrons with energy $\left(E_{S E}\right)$ higher than the applied bias voltage $\left(V_{b}\right)$.

In this study, we apply electronically isolated bias to the specimen by changing electrostatic potentials to suppress the secondary-electrons for atomic imaging. Gold isolated atoms and small clusters on carbon thin film set on the bias holder and both SE and ADF-STEM images are recorded simultaneously. By applying $+10 \mathrm{~V}$ dc on specimen, the image contrast of the cluster labeled " $\mathrm{a}$ " in Figure 2 decreases more than $90 \%$ compared to one without bias. This contrast change is similar to that observed at low magnifications we reported previously. Our study shows for the first time the contrast suppression of the positive dc bias with the TTL detector, suggesting the atom signals are indeed originated from secondary electrons (SE-I) emerged from the specimen surface. Other applications of SE atomic imaging, including semiconductor devices, will be presented. References 
[1] H. Inada, L. Wu, J. Wall, D. Su, \& Y. Zhu, J. of Electron Microsc. 58 (2009) 111-122.

[2] Y. Zhu, H. Inada, K. Nakamura, \& J. Wall, Nature Mater. 8 (2009) 808-812.

[3] C.A. Walsh, Ultramicroscopy 45 (1992) 85-93.
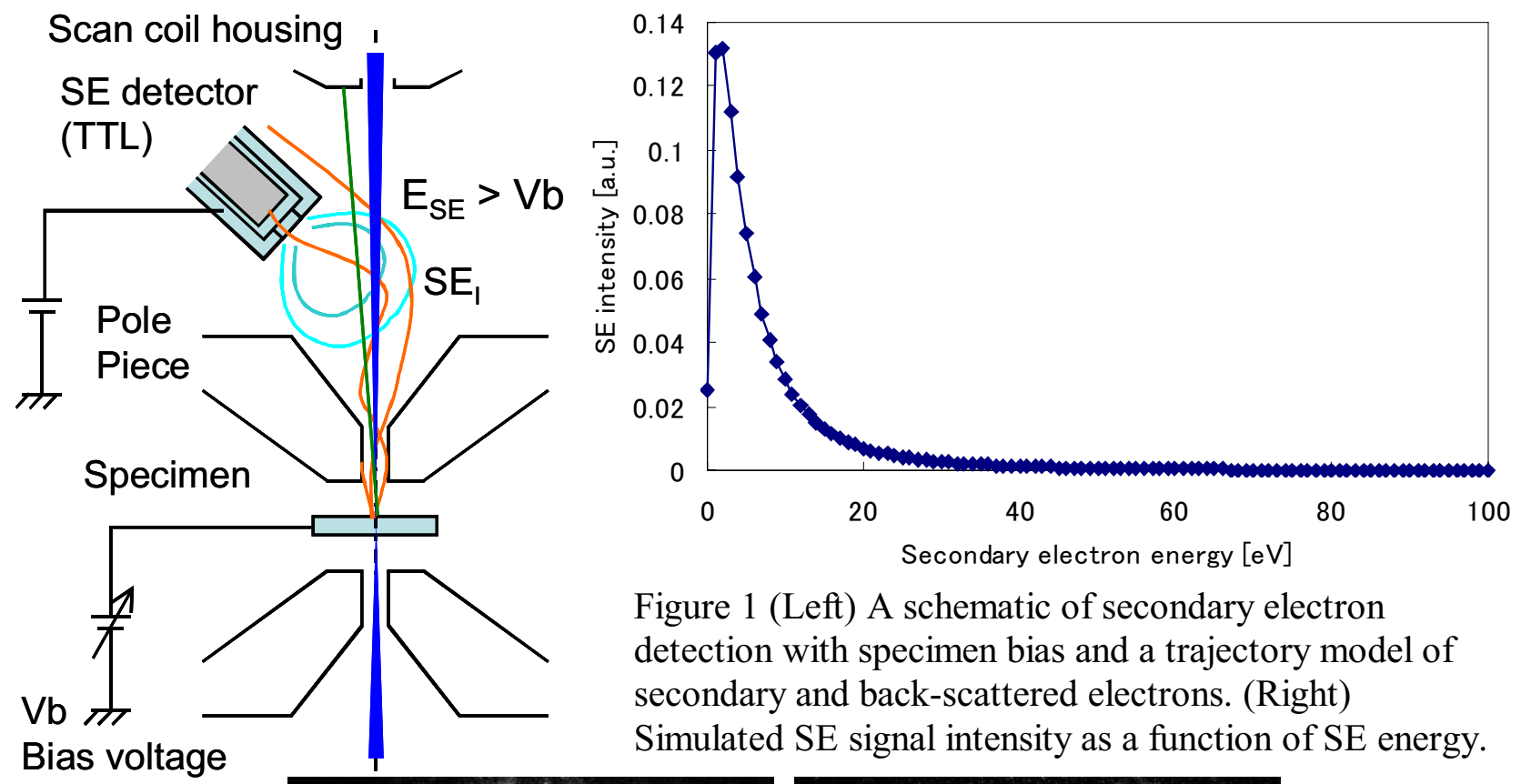

Figure 1 (Left) A schematic of secondary electron detection with specimen bias and a trajectory model of secondary and back-scattered electrons. (Right) Simulated SE signal intensity as a function of SE energy.

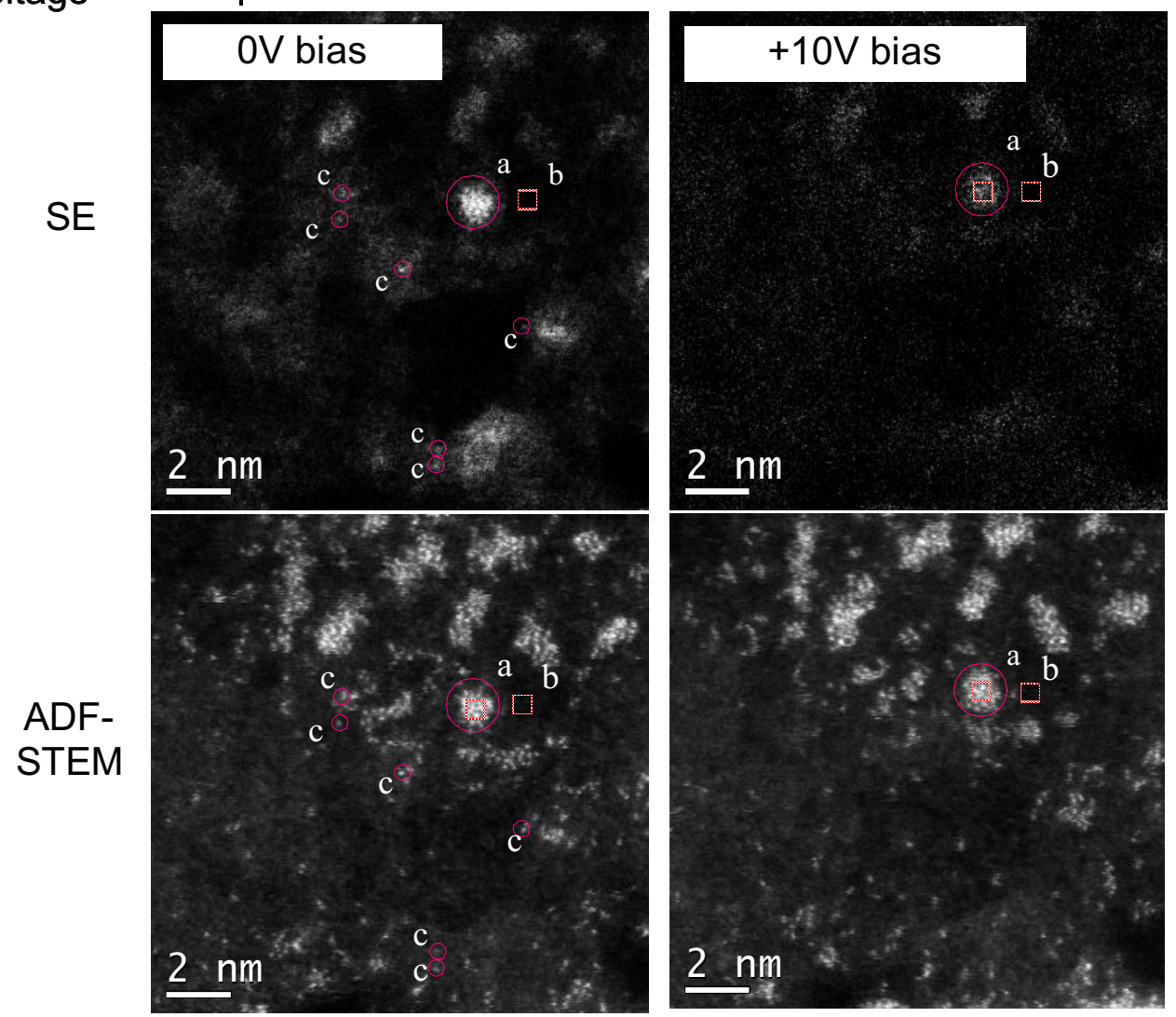

Figure 2. Image comparison of isolated individual gold atoms on thin carbon film with (right) and without (left) applying positive bias. Simultaneous acquired SE images (top row) and ADFSTEM images (bottom row) are shown. 